\title{
Mitochondrially localized PKA reverses mitochondrial pathology and dysfunction in a cellular model of Parkinson's disease
}

\author{
RK Dagda', AM Gusdon ${ }^{1}$, I Pien ${ }^{1}$, S Strack ${ }^{2}$, S Green ${ }^{3}, C$ Li $^{4}$, B Van Houten ${ }^{5}$, SJ Cherra III ${ }^{1}$ and CT Chu ${ }^{\star}{ }^{1,6}$
}

\begin{abstract}
Mutations in PTEN-induced kinase 1 (PINK1) are associated with a familial syndrome related to Parkinson's disease (PD). We previously reported that stable neuroblastoma SH-SY5Y cell lines with reduced expression of endogenous PINK1 exhibit mitochondrial fragmentation, increased mitochondria-derived superoxide, induction of compensatory macroautophagy/ mitophagy and a low level of ongoing cell death. In this study, we investigated the ability of protein kinase A (PKA) to confer protection in this model, focusing on its subcellular targeting. Either: (1) treatment with pharmacological PKA activators; (2) transient expression of a constitutively active form of mitochondria-targeted PKA; or (3) transient expression of wild-type A kinase anchoring protein 1 (AKAP1), a scaffold that targets endogenous PKA to mitochondria, reversed each of the phenotypes attributed to loss of PINK1 in SH-SY5Y cells, and rescued parameters of mitochondrial respiratory dysfunction. Mitochondrial and lysosomal changes in primary cortical neurons derived from PINK1 knockout mice or subjected to PINK1 RNAi were also reversed by the activation of PKA. PKA phosphorylates the rat dynamin-related protein 1 isoform 1 (Drp1) at serine 656 (homologous to human serine 637), inhibiting its pro-fission function. Mimicking phosphorylation of Drp1 recapitulated many of the protective effects of AKAP1/PKA. These data indicate that redirecting endogenous PKA to mitochondria can compensate for deficiencies in PINK1 function, highlighting the importance of compartmentalized signaling networks in mitochondrial quality control.
\end{abstract}

Cell Death and Differentiation (2011) 18, 1914-1923; doi:10.1038/cdd.2011.74; published online 3 June 2011

Parkinson's disease (PD) is an age-related neurodegenerative disease that affects $\sim 1 \%$ of the population in North America. Up to $10 \%$ of PD cases are associated with mutations in PD-linked genes, thought to regulate mitochondrial biology and oxidative-stress response pathways, with effects on ubiquitin proteasome and autophagolysosomal function. ${ }^{1}$ Mitochondrial dysfunction and disruption of mitochondrial homeostasis are implicated in the pathogenesis of both sporadic and genetic forms of PD. ${ }^{1}$

The PARK6 locus encodes for PTEN-induced kinase 1 (PINK1), a putative Ser/Thr kinase that is mutated in an early onset autosomal recessive form of PD that is associated with Lewy body pathology in at least one family. ${ }^{2,3}$ Although the primary functions associated with PINK1 in neurons remain largely unknown, a large body of evidence suggests that PINK1 is cytoprotective. ${ }^{4}$ An engineered kinase-deficient (KD) K219M substitution in the ATP-binding pocket of PINK1 abrogates its ability to protect neurons, ${ }^{5}$ and most PDassociated mutations in PINK1 result in loss of neuroprotective activities. ${ }^{6}$ PINK1 has an $N$-terminal mitochondrial targeting sequence, and loss of PINK1 alters mitochondrial membrane potential and dynamic fission-fusion-based remodeling, while elevating mitochondrial superoxide levels and mitochondrial clearance by macroautophagy. ${ }^{7-10}$

Protein kinase A (PKA) is a tetrameric Ser/Thr kinase with well-defined mechanisms of biological modulation involving differential subcellular targeting by endogenous scaffold proteins. ${ }^{11}$ Outer mitochondrial membrane (OMM) targeting of PKA is achieved by association with three $A$ kinase anchoring proteins (AKAPS). These include (a) AKAP1 or dual specificity kinase AKAP84/121, (b) AKAP2, and (c) Rab32. ${ }^{11}$ PKA can phosphorylate inhibitory serine residues in the pro-apoptotic protein BAD at the OMM, ${ }^{12}$ and also shows inner mitochondrial membrane- or matrix-targeted effects on mitochondrial respiration. ${ }^{13}$

Recently, the fission protein dynamin-related protein 1 (Drp1) has been shown to be reversibly phosphorylated by CDK/Cyclin B, and by PKA or CaM kinase l $\alpha$, at distinct serine residues that border the GTPase exchange domain (GED), with differential effects on fission. ${ }^{14-17}$ The net result of PKAdependent phosphorylation of the rat Drp1 at S656 (equivalent to $\mathbf{S 6 3 7}$ of human Drp1 isoform 1) is suppression of fission, increasing the stability of mitochondrial networks. ${ }^{14,16,18}$ Interestingly, the equivalent serine residue in

\footnotetext{
${ }^{1}$ Department of Pathology, University of Pittsburgh School of Medicine, Pittsburgh, PA, USA; ${ }^{2}$ Department of Pharmacology, University of lowa, lowa City, IA, USA; ${ }^{3}$ Department of Biology, University of lowa, lowa City, IA, USA; ${ }^{4}$ Department of Neurology, Mt. Sinai School of Medicine, New York, NY, USA; ${ }^{5}$ Department of Pharmacology and Chemical Biology, University of Pittsburgh Cancer Institute, University of Pittsburgh School of Medicine, Pittsburgh, PA, USA and ${ }^{6}$ Center for Neuroscience, University of Pittsburgh School of Medicine, Pittsburgh, PA, USA

${ }^{*}$ Corresponding author: CT Chu, Department of Pathology, University of Pittsburgh School of Medicine, 200 Lothrop Street, Pittsburgh, PA 15261, USA.

Tel: 412383 5379; E-mail: ctc4 @ pitt.edu

Keywords: PINK1; PKA; AKAP1; neurodegeneration; mitochondria and mitophagy; 6-hydroxydopamine

Abbreviations: AKAP1, A kinase anchoring protein 1; db-cAMP, dibutyryl cyclic AMP; Drp1, dynamin-related protein 1; MFF, mitochondrial fission/fusion; OMM, outer mitochondrial membrane; PKA, protein kinase A; PINK1, PTEN-induced protein kinase 1; PD, Parkinson's disease; ROS, reactive oxygen species

Received 02.11.10; revised 11.4.11; accepted 30.4.11; Edited by L Scorrano; published online 03.6.11
} 
isoform 3 (S600 of Drp1 isoform 3) is phosphorylated by CaM kinase $l \alpha$, leading to a pro-fission effect, implicating additional modulatory effects of CaM kinase $\mid \alpha$ at other sites. ${ }^{17}$ Calcineurin-dependent dephosphorylation of the PKA site leads to rapid mitochondrial translocation and fission, ${ }^{14,18}$ accounting for the pro-fission effects of PINK1 knockdown in mammalian cells. ${ }^{8}$

In this study, we investigated the ability of mitochondrially targeted PKA, overexpressed or endogenous, to compensate for mitochondrial pathology associated with chronic loss of endogenous PINK1. Our data show that mitochondrial PKA reverses the mitochondrial dysfunction associated with loss of PINK1, implicating involvement in shared or functionally redundant (parallel) mito-protective pathways.

\section{Results}

We previously found that chronic short hairpin RNA (shRNA)mediated knockdown of PINK1 in SH-SY5Y neuroblastoma cells leads to mitochondrial fragmentation, increased mitochondrial superoxide and a compensatory autophagic response that mediates mitochondrial degradation. ${ }^{7}$ Given that AKAP1 functions to target PKA to mitochondria, where it shows mitochondrial stabilizing and cytoprotective functions (reviewed by Carlucci $A$ et al. ${ }^{19}$ ), we hypothesized that increased mitochondrial PKA could rescue parameters of mitochondrial dysfunction induced by impairment of PINK1 function. We tested this hypothesis using pharmacological and molecular methods to modulate the activity or localization of PKA in several clonal SH-SY5Y cell lines (D14, A14, and A4) with stable $50-90 \%$ reductions in PINK1 expression compared with a line stably expressing the control shRNA vector (V17) (Supplementary Figure 1a). ${ }^{7}$

Mitochondrially targeted PKA reduces mitochondrial fragmentation in PINK1-deficient cells. Treating the PINK1 shRNA lines with pharmacological activators of PKA, forskolin and dibutyryl-cyclic AMP (db-cAMP) $\times 4 \mathrm{~h}$ reversed mitochondrial fragmentation induced by stable loss of PINK1 (Figures 1a and b; Supplementary Figure 5a). To study whether or not mitochondrially targeted PKA is involved in these effects, PINK1 shRNA lines were transfected with OMM-GFP as control, OMM-targeted PKA (Supplementary Figure 1b), or untargeted PKA, giving rise to similar levels of recombinant PKA expression (Figure 1c), and eliciting similar levels of PKA activity as measured in cells expressing a CRE-luciferase reporter (Supplementary Figure 1c). In the presence of OMM-PKA, PINK1 shRNA lines no longer showed decreased interconnectivity compared with the vector control line (Figure 1d; Supplementary Figure 5b).

As overexpression of OMM-PKA may also show a basal effect in the control line, we tested whether redirecting an endogenous pool of cytosolic PKA to mitochondria using AKAP1 has a similar effect. Indeed, transient expression of the wild-type PKA-targeting core domain of AKAP1 also reversed mitochondrial fragmentation in each of the three PINK1 shRNA lines. Importantly, a point mutant of AKAP1 (AKAP1- $\triangle P K A$ ) that fails to bind PKA, but still localizes to mitochondria $^{12}$ (Supplementary Figure $1 \mathrm{~b}$ ), was unable to rescue mitochondrial fragmentation, supporting a specific role for mitochondrially targeted PKA in reversing this effect of PINK1 deficiency (Figure 1d). The ability of AKAP1 to reverse mitochondrial fragmentation in PINK1 shRNA cells is inhibited by H89, a pharmacological inhibitor of PKA activity (Figure 1e).

As reported, ${ }^{7}$ electron micrographs of PINK1 shRNA cell lines reveal increased numbers of lysosomes and autophagic vacuoles (AV) (Supplementary Figure 2a). Transfection with AKAP1 reversed these changes (Supplementary Figures $2 a$ and b). PINK1 shRNA cells expressing OMM-PKA also showed decreased lysosomal content, but additionally showed perinuclear clustering of mitochondria (Supplementary Figure 2a; bottom right). Although overexpression of OMM-PKA showed additional effects compared with AKAP1mediated mitochondrial redistribution of endogenous PKA, both methods reversed mitochondrial fragmentation in PINK1 shRNA lines, whereas overexpression of untargeted PKA did not (Figure 1d).

PKA or AKAP1 reduces the autophagic response in PINK1-deficient cells. We have previously established that compensatory autophagy and mitochondrial degradation is triggered in response to increased mitochondrial reactive oxygen species (ROS) in these PINK1 shRNA lines., ${ }^{7,20}$ Pharmacological activation of PKA signaling suppressed the increase in GFP-LC3-labeled AVs in these PINK1-deficient cells (Figure 2a; Supplementary Figure $5 \mathrm{c}$ ). To investigate the effects of GFP-tagged constructs that direct PKA to different subcellular compartments, cells were co-transfected with RFP-LC3, which labels both early and acidified AVs. ${ }^{21}$ Transient expression of PKA, OMM-PKA, or AKAP1, but not mutant AKAP1, suppressed the increase in RFP-LC3 puncta per cell when compared with OMM-GFP-transfected PINK1 shRNA cells (Figure 2b; Supplementary Figure $5 \mathrm{~d}$ ). Treating cells with $\mathrm{H} 89$ blocked the ability of AKAP1 to reduce AVs in PINK1 shRNA cells (Figure 2c), confirming involvement of endogenous PKA.

To examine the effects on mitophagy, we transiently transfected cells with the mitochondrially targeted GFPtagged PKA modulating constructs, and measured colocalization with RFP-LC3 puncta as previously described. ${ }^{7}$ Confocal microscopy analyses confirmed that stable knockdown of PINK1 increased the presence of mitochondria in AVs (Figure 2d). Separate experiments confirmed increased delivery of mitochondria to Lysotracker Red (Molecular Probes, Eugene, CA, USA) stained lysosomes, indicative of AV maturation (data not shown). We found that either OMMPKA or AKAP1 suppressed the increased mitochondrial delivery to RFP-LC3-labeled AVs (Figure 2d; supplementary Figure 5e) in PINK1 shRNA cell lines. Single-cell analysis of mitochondrial content in cells transiently co-expressing a mito-RFP and the GFP-tagged PKA modulators show partial restoration of mitochondrial content in PINK1 shRNA lines (Supplementary Figure 3 ). In this system, there were no effects of OMM-PKA or of AKAP1 on levels of mitochondrial transcription factor $A$, a central regulator of mitochondrial biogenesis (data not shown), and the AKAP1 construct used lacks the $C$-terminal-binding domains for src and mRNA involved in regulating mitochondrial biogenesis. ${ }^{22}$ As the loss 
a

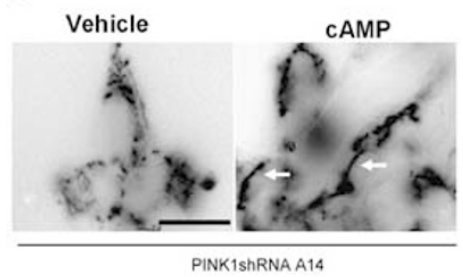

b
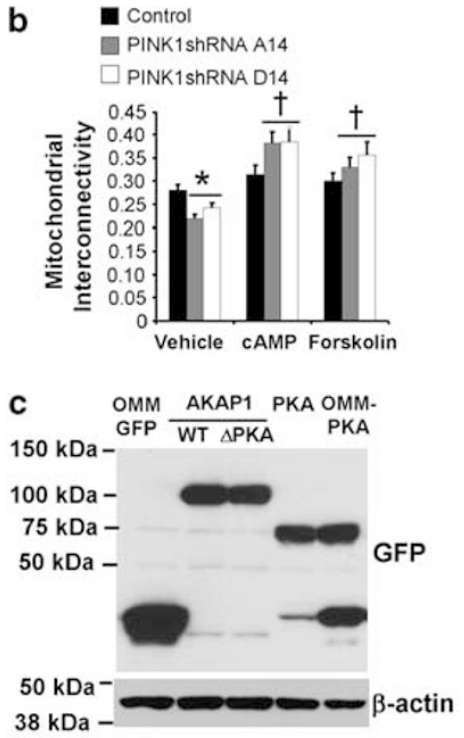

d

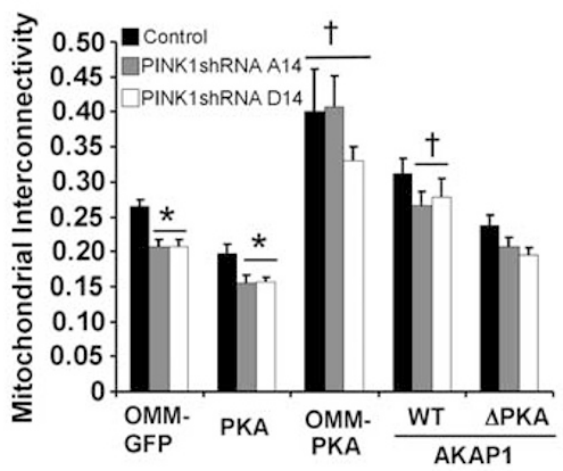

e

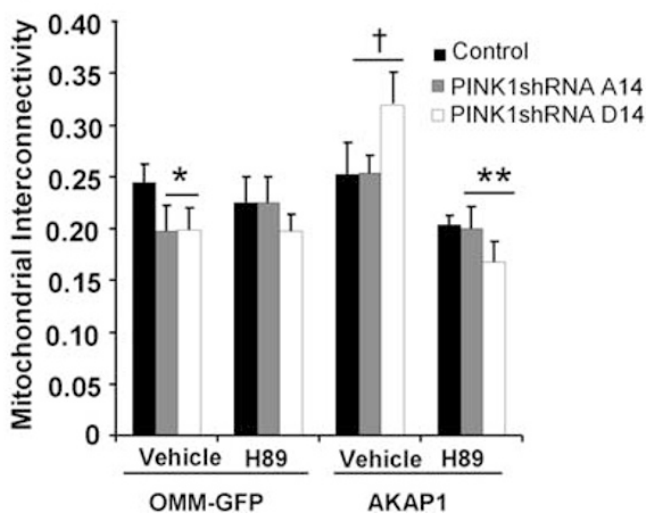

Figure 1 Increased mitochondrial PKA reverses mitochondrial fragmentation in PINK1-knockdown SH-SY5Y cells. (a) Epifluorescence images of OMM-GFP-expressing mitochondria in stable PINK1 shRNA line A14 in the presence or absence of db-cAMP $(250 \mu \mathrm{M})$. White arrows point to examples of fused mitochondria. Scale bar: $20 \mu \mathrm{m}$. (b) Quantification of mitochondrial interconnectivity in vector stable control and PINK1 shRNA clonal lines in the presence or absence of PKA activators (db-cAMP and forskolin), or water as vehicle control (compiled means \pm S.E.M., $n=3$ independent experiments; ${ }^{*} P<0.05$ versus vehicle/control; $\dagger P<0.05$ versus respective vehicle/ PINK1 shRNA line, ANOVA). (c) Lysates from cells transiently expressing the indicated GFP-tagged PKA or AKAP1 constructs were immunoblotted for GFP to analyze expression levels. (d) Quantification of mitochondrial interconnectivity in control and PINK1 shRNA lines transiently co-expressing the indicated panel of GFP-tagged fusion constructs of PKA-modulating plasmids (compiled means \pm S.E.M., $n=5$ independent experiments; ${ }^{*} P<0.05$ versus respective transfected control cell line; $† P<0.05$ versus respective OMM-GFP-transfected cell line, ANOVA). (e) Quantification of mitochondrial interconnectivity in control or PINK1 shRNA clonal cell lines transiently coexpressing OMM-GFP or AKAP1 in the presence or absence of the PKA inhibitor $\mathrm{H} 89(0.5 \mu \mathrm{M} \times 4 \mathrm{~h}$; a dose that does not affect mitochondrial interconnectivity in control PINK1 shRNA cells) (means \pm S.E., 20-35 cells/condition, two independent experiments; ${ }^{*} P<0.05$ versus OMM-GFP/vehicle/control cell line; $\dagger P<0.05$ versus OMM-GFP/ vehicle/respective PINK1 shRNA line; ${ }^{*} P<0.05$ versus AKAP1-expressing/vehicle-treated/respective PINK1 shRNA line, ANOVA)

of mitochondrial content observed in PINK1 shRNA cells can be reversed by inhibiting autolysosomal degradation, ${ }^{7}$ these data indicate that redirecting endogenous levels of PKA to mitochondria is sufficient to reverse changes in mitochondrial fragmentation and autophagolysosomal degradation observed in PINK1 shRNA stable cell lines.

Chronic loss of PINK1 leads to mitochondrial functional deficits reversed by AKAP1 or OMM-PKA. Given that the autolysosomal response is often triggered in response to mitochondrial dysfunction and $\mathrm{ROS},{ }^{23}$ we investigated whether enhanced mitochondrial PKA targeting could correct functional deficits observed in PINK1-deficient cells. ${ }^{7,9,24}$ As expected, PINK1 deficiency was associated with a $27-35 \%$ decrease in transmembrane potential and increased MitoSOX oxidation, ${ }^{7,9}$ both of which were reversed by enhanced PKA signaling (Figures $3 a$ and b). Given the caveats associated with fluorescent ROS indicators, we confirmed these results by transfection with a mitochondrially targeted bacterial hydrogen peroxide-sensing regulatory domain (mito-HyPer) (Supplementary Figure 4).

Expanding upon previous studies that showed that endogenous PINK1 has a role in regulating mitochondrial respiration, ${ }^{25}$ we found that loss of PINK1 decreased both basal mitochondrial respiration and maximal uncoupled respiration, as measured in live cells using a Seahorse Extracellular Flux Analyzer (Seahorse Bioscience, North Billerica, MA, USA) (Figure 3c). Moreover, either db-cAMP treatment or expression of OMM-PKA or AKAP1 increased the total mitochondrial respiratory capacity (MRC: the FCCPinduced maximal oxygen consumption rate (OCR) minus residual OCR in presence of rotenone) compared with PINK1 shRNA D14 cells transiently expressing GFP alone (Figure 3d). OMM-PKA also increased basal OCR and MRC in PINK1 shRNA clone A14 (Supplementary Figure 6a). When grown in glucose-free, galactose-containing media, SH-SY5Y cells are dependent on mitochondrial respiration for ATP generation. ${ }^{26}$ In further support of a role for AKAP1/PKA in 

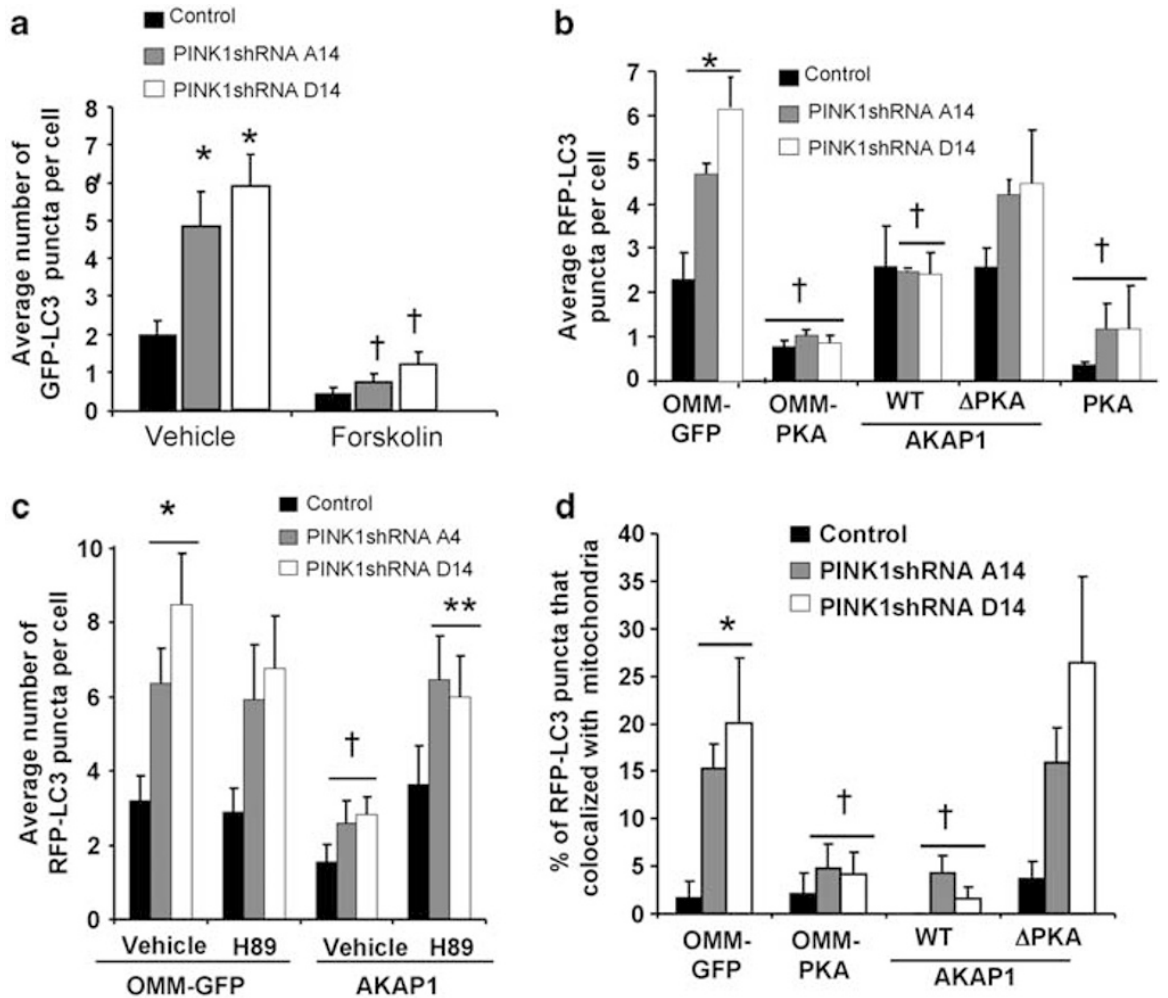

Figure 2 Macroautophagy and mitophagy elicited by loss of endogenous PINK1 is reversed by mitochondrially localized PKA. (a) The average number of GFP-LC3 puncta per cell in control and PINK1 shRNA clonal cell lines treated with forskolin or DMSO as a vehicle control (compiled means \pm S.E.M, $n=5$ experiments, 15-25 cells per condition per experiment; * $P<0.05$ versus vehicle/control; ${ }^{\dagger} P<0.05$ versus respective vehicle/PINK1 shRNA line, one-way ANOVA). (b) The average RFP-LC3 puncta per cell in control and PINK1 shRNA clonal lines co-expressing the indicated GFP-tagged constructs with RFP-LC3 as a marker of AVs (compiled means \pm S.E.M, $n=5$ experiments, $15-25$ cells per condition per experiment; ${ }^{*} P<0.05$ versus OMM-GFP/control cell line; ${ }^{\dagger} P<0.05$ versus respective OMM-GFP-transfected cell lines, ANOVA). (c) Quantification of RFP-LC3 puncta/cell in control or PINK1 shRNA lines transiently co-expressing OMM-GFP or AKAP1-GFP in the presence or absence of the PKA inhibitor H89 $(0.5 \mu \mathrm{M} \times 4 \mathrm{~h})\left(\right.$ mean $\pm \mathrm{S}$.E, $20-30$ cells/condition, two independent experiments; ${ }^{*} P<0.05$ versus OMM-GFP/vehicle/control line; ${ }^{\dagger} P<0.05$ versus OMM-GFP/vehicle/ respective PINK1shRNA line; ${ }^{* *} P<0.05$ versus AKAP1-expressing/vehicle/respective PINK1 shRNA line, ANOVA). (d) Quantitative analysis of RFP-LC3 colocalization with mitochondria as an index of mitochondrial delivery to AVs in clonal vector control and PINK1 shRNA lines expressing the indicated mitochondrially localized GFP constructs (means \pm S.E., $25-35$ cells/condition, three independent experiments; ${ }^{\star} P<0.05$ versus OMM-GFP/control; ${ }^{\dagger} P<0.05$ versus respective OMM-GFP/PINK1 shRNA cell line, ANOVA)

reversing mitochondrial dysfunction in PINK1-knockdown cells, $48 \mathrm{~h}$ of AKAP1 transient expression significantly increased steady-state levels of mitochondrially derived ATP in stable PINK1-knockdown lines grown in glucose-free media (Figure 3e; Supplementary Figure 6b).

To determine whether the effects of mitochondrial PKA are observed in other parkinsonian models exhibiting mitochondrial fragmentation and autophagy, we studied the effects of untargeted or targeted constructs in the 6-hydroxydopamine injury model. Similar to the PINK1-deficient model, only mitochondrially targeted PKA or AKAP1 could reverse effects on mitochondrial fragmentation (Supplementary Figure 7a), but either untargeted or targeted PKA could suppress the autophagy response (Supplementary Figure $7 \mathrm{~b}$ ) induced by 6-hydroxydopamine $(120 \mu \mathrm{M}$ dose $\times 4 \mathrm{~h})$.

To investigate whether the reversal in mitochondrial dysfunction and oxidative stress mediated by AKAP1/PKA affects viability of PINK1 shRNA cell lines, we studied the effects of transiently transfecting AKAP1 and OMM-PKA on cell death induced by loss of PINK1. In brief, we found that transient expression of wild-type AKAP1 and OMM-PKA, but not AKAP1- $\triangle \mathrm{PKA}$ or untargeted PKA, blocked the increased rate of apoptosis observed in PINK1-deficient cells, as assessed by the percentage of GFP-expressing cells that show fragmented/pyknotic nuclei or that exhibit diffuse cytochrome $c$ distribution (Figure 4; Supplementary Figure 8).

Mitochondrial pathology associated with PINK1 deficiency in primary neurons is reversed by activation of PKA. To determine whether loss of endogenous PINK1 in primary cortical neurons recapitulates mitochondrial and lysosomal pathology seen in PINK1 shRNA SH-SY5Y cell lines, primary cortical neurons were isolated from wild-type, heterozygous and homozygous PINK1-knockout embryonic mice (Figure $5 \mathrm{a}$ ), or wild-type primary cortical neurons were transiently transfected with a PINK1 short interfering doublestranded RNA, which efficiently reduces the endogenous protein levels of murine PINK1 in HT22 cells (Figure 5e).

We observed that homozygous PINK1-knockout primary cortical neurons showed significantly decreased mitochondrial interconnectivity (Figure $5 \mathrm{~b}$ ), reduced membrane potential as assessed by TMRM staining (Figure $5 \mathrm{c}$ ), and lysosomal 


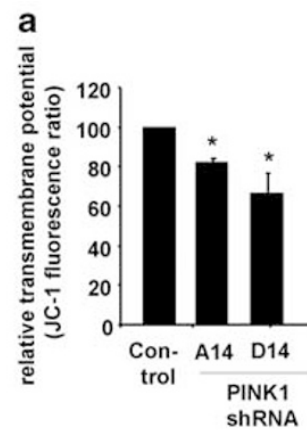

C

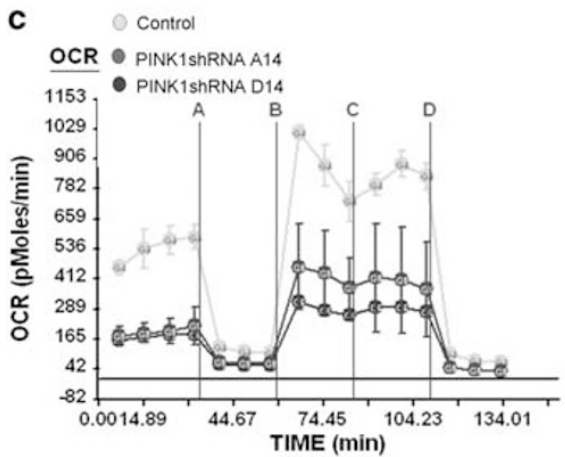

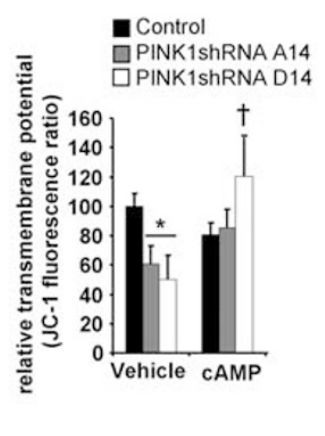

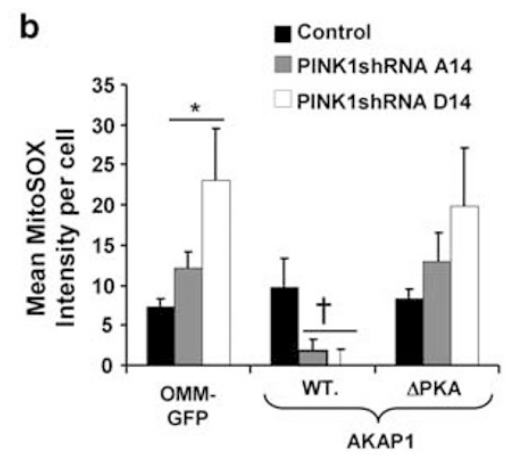

d

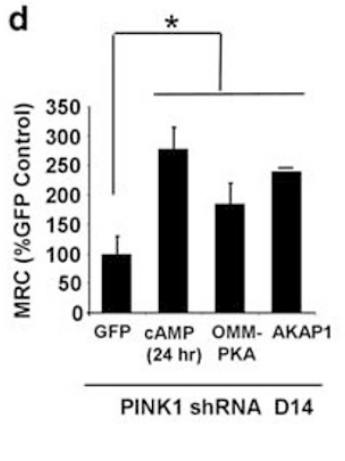

e

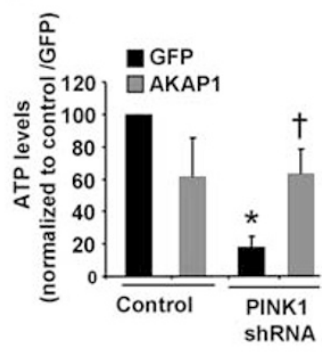

Figure 3 Mitochondrial dysfunction in PINK1-deficient cells is reversed by AKAP1 or OMM-PKA. (a) Fluorescence JC-1 assay showing that PINK1 shRNA clonal cell lines have significantly reduced transmembrane potentials (left; compiled means \pm S.E.M, $n=3$ independent experiments). Administering db-cAMP $(250 \mu \mathrm{M} \times 4 \mathrm{~h})$ reverses the mitochondrial membrane potential decreases in PINK1 shRNA lines (right; means \pm S.E., eight wells, three independent experiments; ${ }^{\star} P<0.05$ versus control cell line/ vehicle; ${ }^{\dagger} P<0.05$ versus respective vehicle-treated PINK1 shRNA clonal line, ANOVA). (b) MitoSOX intensity in the indicated cell lines transiently expressing the indicated forms of AKAP1 or OMM-GFP (means \pm S.E, $40-50$ cells/condition, four experiments; ${ }^{*} P<0.05$ versus OMM-GFP/control cell line; ${ }^{\dagger} P<0.05$ versus respective OMM-GFP/ PINK1 shRNA cell line, ANOVA). (c) Representative Seahorse trace of two experiments showing live-cell OCRs for vector-stable control and two PINK1 shRNA cell lines, sequentially treated with oligomycin (a), FCCP (b), 2-DG (c), and rotenone (d) to delineate basal, total mitochondrial respiratory capacity (MRC = FCCP minus rotenone), and mitochondria-independent oxygen consumption. (d) The effects of db-cAMP and transient expression of OMM-PKA or AKAP1 on total MRC in PINK1 shRNA line D14, which shows the most robust knockdown of PINK1 (Supplementary Figure 1a) $\left({ }^{*} P<0.05\right.$ versus GFP transfected). (e) Mitochondria-dependent ATP levels were quantified in the control stable V17 and the PINK1 shRNA D14 line expressing either GFP or AKAP1 and grown in galactose/glutamine media to suppress glycolysis. Mitochondrial complex inhibitors reveal that $>70 \%$ of ATP levels are derived from mitochondria under these conditions (compiled means, \pm S.E.M, $n=3$ experiments; ${ }^{*} P<0.05$ versus vector control cell line/ GFP; ${ }^{\dagger} P<0.05$ versus PINK1 shRNA/GFP, ANOVA)
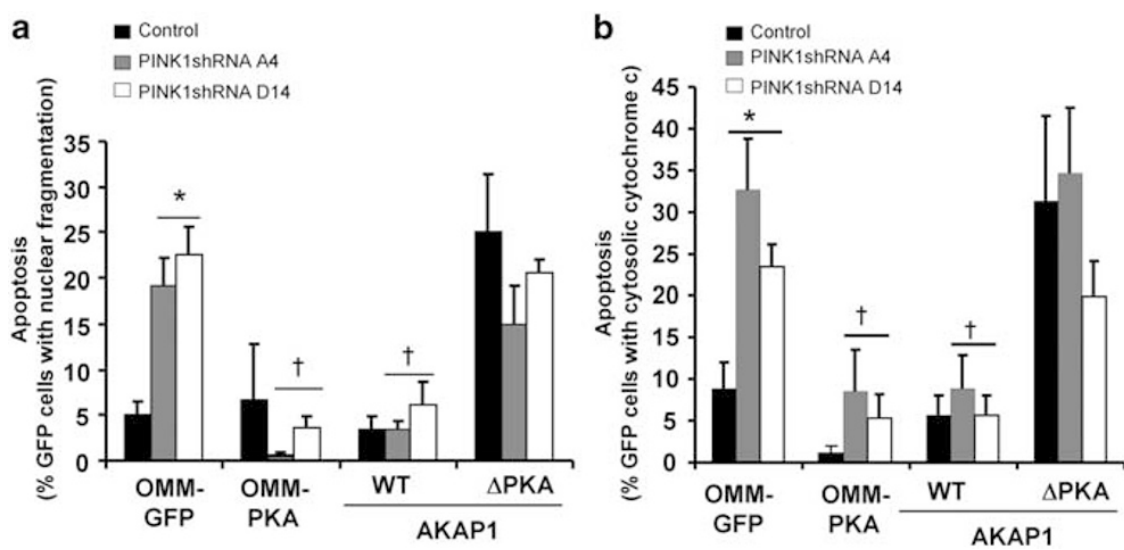

Figure 4 Cell death induced by loss of PINK1 is reversed by AKAP1 or OMM-PKA. Control and PINK1 shRNA cells were transiently transfected with OMM-GFP or the indicated GFP-tagged fusion constructs for $48 \mathrm{~h}$ and quantified for cell death using two different methods. (a) Cell death was determined by quantifying the percentage of GFPpositive cells that exhibit apoptotic DAPI-stained nuclei (fragmented or pyknotic) per epifluorescence micrograph field. (b) Cell death was determined by quantifying the percentage of GFP-positive cells containing cytosolic cytochrome $c$ staining (compiled means \pm S.E.M., $n=4$ representative experiments; ${ }^{*} P<0.05$ versus OMM-GFP/ control; ${ }^{\dagger} P<0.05$ versus respective OMM-GFP/ PINK1 shRNA cell line, ANOVA)

expansion as assessed by Lysotracker Red (Figure 5d) compared with wild-type primary cortical neurons, each of which was completely reversed by db-cAMP $\times 4 \mathrm{~h}$. Similar results were observed in response to transient RNAimediated knockdown of PINK1 in primary cortical neurons (Figures 5f-g). 


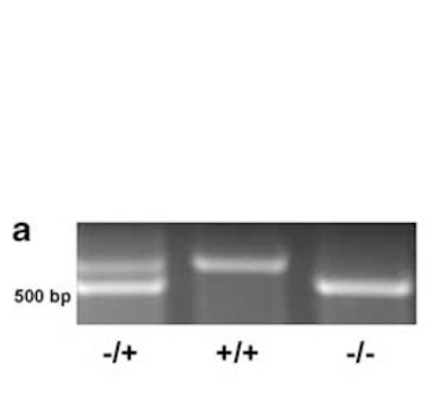

c

Vehicle

CAMP

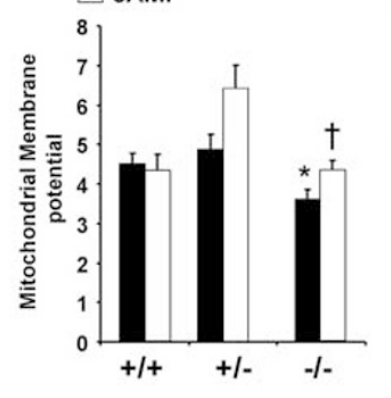

b

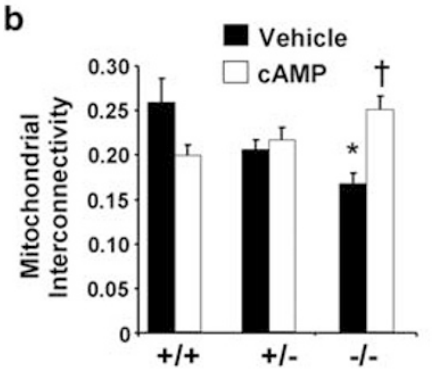

d

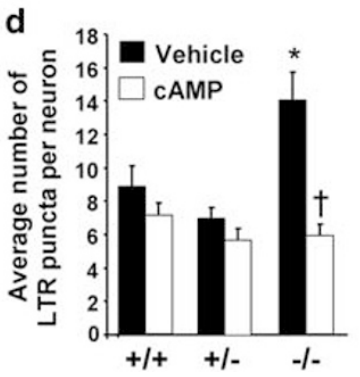

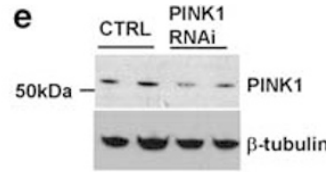

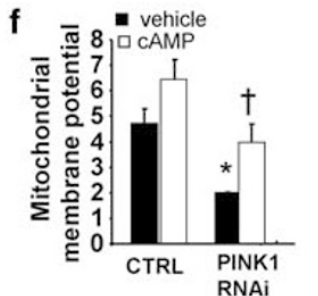

g

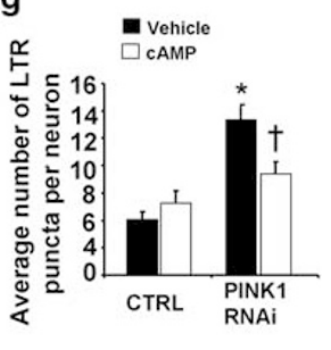

Figure 5 Treatment with db-cAMP reverses mitochondrial injury and lysosomal expansion induced by loss of endogenous PINK1 in primary cortical neurons. (a) Prenatal E14 mouse pups from mating of PINK1 $+/$ - mice were genotyped by PCR amplification. Heterozygous mouse pups show the presence of two bands at 510 and 630 bp, whereas KO pups only show the lower PCR product. (b) Quantification of mitochondrial interconnectivity in wild-type, heterozygous, and PINK1-knockout cortical neurons after $4 \mathrm{~h}$ with vehicle or $250 \mu \mathrm{M}$ db-cAMP (means \pm S.E. from three independent experiments, $20-25$ neurons/condition/experiment; ${ }^{*} P<0.05$ versus wild-type/vehicle; ${ }^{\dagger} P<0.05$ versus PINK1 homozygous knockout/vehicle, ANOVA). (c) Quantification of TMRM mitochondrial membrane potential in PINK1-knockout cortical neurons (means \pm S.E., from two independent experiments, $30-35$ neurons/condition; ${ }^{*} P<0.05$ versus wild-type/vehicle; ${ }^{\dagger} P<0.05$ versus PINK1 homozygous knockout/vehicle, ANOVA). (d) The average number of LTR-stained lysosomes per neuron (means \pm S.E. from three independent experiments; ${ }^{\star} P<0.05$ versus wild-type/vehicle; ${ }^{\dagger} P<0.05$ versus PINK1 homozygous knockout/vehicle, ANOVA). (e) Western blot analysis of endogenous PINK1 levels in cell lysates derived from HT22 cells transiently transfected with PINK1 siRNA for 3 days. (f) Quantification of the mitochondrial membrane potential in cortical neurons transfected with PINK1 siRNA (means \pm S.E., two independent experiments, 30-35 neurons/condition; ${ }^{*} P<0.05$ versus control siRNA/vehicle; ${ }^{\dagger} P<0.05$ versus $P I N K 1$ siRNA/vehicle, ANOVA). (g) The average number of lysosomes per cortical neuron treated with control or PINK1 siRNA in the presence or absence of db-cAMP (means \pm S.E., two independent experiments, $20-25$ neurons/condition; ${ }^{*} P<0.05$ versus control siRNA/vehicle treated; ${ }^{\dagger} P<0.05$ versus PINK1 siRNA/vehicle treated, ANOVA)

The pro-survival effects of AKAP1/PKA in PINK1 shRNA cell lines can be mimicked by PKA-mediated phosphorylation of Drp1. As stable loss of PINK1 leads to calcineurin activation ${ }^{8}$ and Drp1-dependent mitochondrial fragmentation, ${ }^{7}$ which occurs upstream of macroautophagy/ mitophagy, ${ }^{7,27}$ we investigated whether the effects of mitochondrially targeted PKA might converge on Drp1 regulation. The fission activity of Drp1 is suppressed by PKA-mediated phosphorylation. ${ }^{14,16}$ Using an antibody raised against the PKA phosphorylation site of rat Drp1 (S656), ${ }^{14}$ we verified that transient expression of OMM-PKA in SH-SY5Y cells increased phosphorylation of endogenous Drp1, with AKAP1/cAMP-mediating a smaller response (Supplementary Figures $9 \mathrm{a}$ and $\mathrm{b}$ ), consistent with the stronger basal effects of OMM-PKA observed earlier.

We used engineered mutations of rat Drp1 at the PKA site (S656 in rat Drp1 isoform 1) to assess its role in the protective effects of PKA modulation in PINK1-deficient cells. The PKA phosphorylation resistant (S656A) and phosphomimetic (S656D) Drp1 mutants were co-transfected with mito-RFP or RFP-LC3 to analyze for mitochondrial morphology and autophagy, respectively. These previously characterized mutant Drp1 plasmids are designed with shRNA to efficiently silence endogenous Drp1 while expressing RNAi-resistant Drp1 mutants. ${ }^{14}$ Transient expression of Drp1-(S656D) is sufficient to increase mitochondrial interconnectivity and block induction of macroautophagy induced by loss of PINK1, whereas transient expression with the non-phosphorylatable S656A mutation did not show these protective effects (Figures $6 a$ and b). Furthermore, the suppressive effects of AKAP1 on mitochondrial fragmentation and autophagy involves Drp1, as replacement of cellular Drp1 with a non-phosphorylable S656A mutant reduces the ability of AKAP1 or db-cAMP to reverse these effects in PINK1 shRNA cells (Supplementary Figures $9 \mathrm{c}$ and $\mathrm{d}$ ). These results indicate that phosphorylation of Drp1 at this site contributes to the protective effects of enhanced mitochondrial PKA signaling.

Finally, transient expression of Drp1-(S656D), but not the non-phosphorylatable S656A, inhibited the increased cell death observed in stable PINK1 shRNA cell lines (Figures 6c and d). In aggregate, these results indicate that mitochondrial targeting of PKA reverses mitochondrial dysfunction, fragmentation, autophagy, and cell death induced by loss of PINK1 function, and that PKA-mediated phosphorylation of Drp1 is sufficient to stabilize mitochondrial networks and promote cell survival in PINK1 shRNA lines. 
a

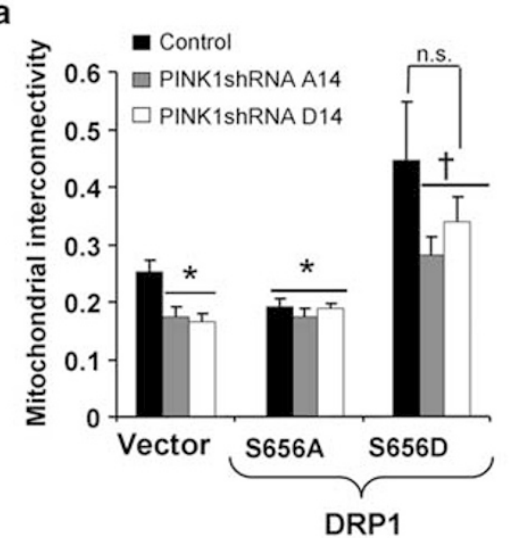

c

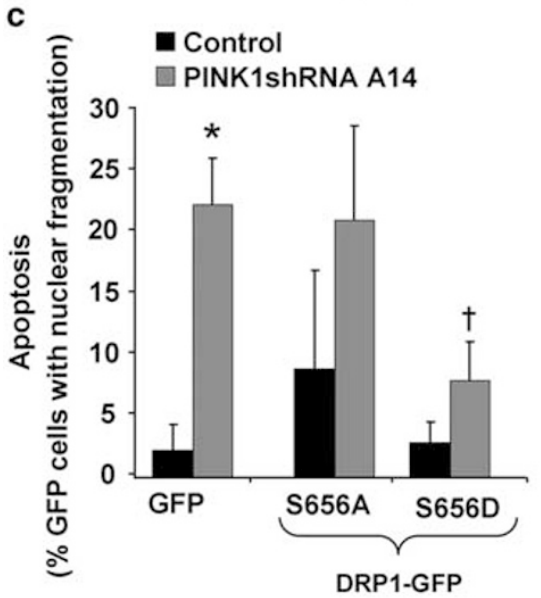

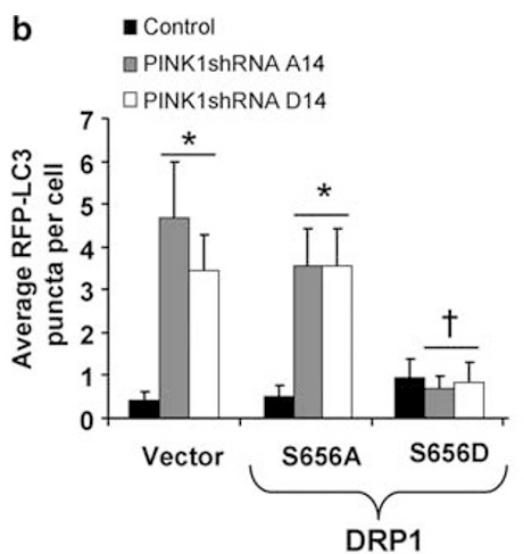

d

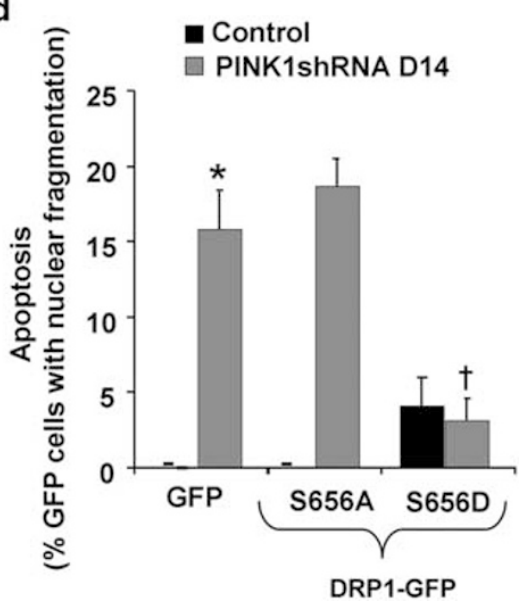

Figure 6 The effects of AKAP1/OMM-PKA on mitochondrial fission, autophagy and survival are reproduced by mimicking PKA-mediated phosphorylation of Drp1. (a) Quantification of mitochondrial interconnectivity in control and PINK1 shRNA lines co-expressing mito-RFP to label mitochondria and the indicated GFP-tagged Drp1 forms. Phosphorylation at S656 inactivates fission activity of Drp1, mimicked by the S656D mutation. The non-phosphorylatable S656A mutant is fission active (means \pm S.E, 20-35 cells/condition, three independent experiments; ${ }^{*} P<0.05$ versus vector/control; ${ }^{\dagger} P<0.05$ versus vector/PINK1 shRNA; n.s. $=$ not significant, ANOVA). (b) The effects of the indicated GFP-tagged Drp1 constructs on RFP-LC3 puncta in the indicated cell lines (means \pm S.E, $20-40$ cells/condition, three independent experiments; ${ }^{*} P<0.05$ versus vector/control cell line; $\dagger P<0.05$ versus vector/PINK1 shRNA cell line, ANOVA,). (c and d) Control and PINK1 shRNA cell lines were transiently transfected with GFP as a control or the indicated GFP-tagged Drp1 mutants for $48 \mathrm{~h}$. Cell death was determined by quantifying the percentage of GFP-positive cells that exhibit apoptotic DAPIstained nuclei (fragmented or pyknotic) per epifluorescence micrograph field as shown for PINK1 shRNA A14 and for PINK1 shRNA D14 (means \pm S.E., 150-200 cells/ condition, three independent experiments; ${ }^{*} P<0.05$ versus GFP/control; ${ }^{\dagger} P<0.05$ versus GFP/PINK1 shRNA line ANOVA)

\section{Discussion}

Although our understanding of neuronal pro-survival signaling pathways has significantly increased over the past two decades, there are currently no therapies to slow disease progression in neurodegenerative diseases. Although neuroprotective transcriptional responses may be reduced in $\mathrm{PD},{ }^{28,29}$ neuroprotection can also be mediated through nontranscriptional mechanisms. In particular, a key role for mitochondrial kinase networks in stress responses has been emerging. ${ }^{4}$ In this report, we found that redirecting endogenous PKA to mitochondria via AKAP1, or simply overexpressing mitochondrially targeted PKA (OMM-PKA), rescues mitochondrial and lysosomal changes manifested in PINK1 shRNA cell lines. Mitochondrial depolarization and lysosomal changes were likewise observed in two PINK1-deficient primary neuron systems, with reversal by db-cAMP. Mitochondrially targeted PKA reversed several parameters of mitochondrial dysfunction, including partial rescue of mitochondrially derived ATP, while opposing the fissioninducing effects of calcineurin ${ }^{8}$ in PINK1-knockdown cells.

The neuroprotective role of AKAP1 has traditionally been thought to involve in modulation of apoptosis. ${ }^{12}$ The current data extend the protective effects of AKAP1 to include upstream mechanisms involving restoration of mitochondrial respiratory function (Figure 3 ), reduction of mitochondrial ROS and reduction of mitochondrial fission (Figure 1). Although AKAP1 is a multi-domain protein that interacts with the tyrosine phosphatase PTPD1, the tyrosine kinase Src, as well as directing mRNA to the OMM, ${ }^{11,12,22}$ the AKAP1 construct used in this study only recruits PKA to the mitochondria (amino acids 1-524). Moreover, H89 reversed the effects of AKAP1, an AKAP1 point mutant unable to bind PKA was ineffective, and transfection with OMM-targeted PKA exhibited similar effects. These indicate that protective effects of AKAP1 were mediated through PKA.

Transient expression of the PKA phospho-mimetic mutant (S656D) ${ }^{14}$ was sufficient to recapitulate the effects of AKAP1 
in ameliorating mitochondrial pathology and reducing neuronal cell death in the setting of abnormal PINK1 function (Figure 6). OMM-targeting of PKA was needed for modulating mitochondrial morphology, function, and cell death in the PINK1-deficient context. These data suggest that AKAP1 exerts its mitochondrial stabilizing effects through localized targeting of PKA catalytic activity to the OMM, resulting in PKA-mediated phosphorylation of Drp1 in PINK1 shRNA cells (Supplementary Figure 9). Although S656D reduces mitochondrial fission activity in Drp1 isoform 1, the precise roles of GTPase activity, altered localization, or other changes in protein interactions, with regard to its ability to reduce cell death, remain to be clarified given differences reported in the literature. ${ }^{14,16-18}$

Potentially distinct pools of PINK1 and PKA seem to regulate macroautophagy versus mitochondrial function in neuronal cells. Although only mitochondrially targeted PKA was able to compensate for the mitochondrial defects of PINK1 deficiency (respiration, membrane potential, ROS, fission-fusion, mitophagy), either untargeted or mitochondrially targeted PKA was able to suppress autophagy. Similar patterns were seen with 6-hydroxydopamine injury. The effects of PKA on general autophagy may relate to its recently described activity in phosphorylating the autophagy protein LC3 in the cytosol. ${ }^{30}$ Likewise, transient overexpression of a RNAi-resistant $N$-terminal deletion mutant of PINK1 that is impaired for mitochondrial targeting suppresses macroautophagy in PINK1 shRNA cells, ${ }^{7}$ while mitochondrial targeting is necessary for the ability of overexpressed PINK1 to prevent MG132-induced cytochromc $c$ release. ${ }^{6}$ Interestingly, alterations in the processing of PINK1 seem to regulate its activities in stabilizing mitochondrial function ${ }^{31}$ versus its ability to recruit parkin to depolarized mitochondria, ${ }^{32}$ and decreased processed/full-length ratios suggested to mediate mitochondrial dysfunction and clearance. ${ }^{31}$ In any case, the observation that cell death was reduced by PKA manipulations that also rescued mitochondrial parameters is compatible with a compensatory, rather than pathogenic, role for autophagy in PINK1-deficient cells. ${ }^{7}$

Interestingly, mitochondrial PKA not only reversed fission and autophagy, as predicted by its ability to phosphorylate Drp1, LC3, and Atg13, but also significantly improved mitochondrial function in PINK1-deficient cells. Loss of endogenous PINK1 has been shown to cause changes in mitochondrial structure, decreased substrate utilization at complex I and IV, and decreased transmembrane potential. $^{7,24,25,33}$ Our results show that re-directing endogenous PKA to the mitochondria improved maximal respiration in PINK1-deficient cells, suppressing reactive-oxygen species production, and supporting mitochondrially derived ATP production. As AKAP1 can also enhance mitochondrial function and biogenesis, ${ }^{19}$ and PKA promotes phosphorylation of complex I components, ${ }^{13}$ the mitoprotective effects of localized PKA signaling may involve several mechanisms.

Both PKA and PINK1 can increase the stability and function of mitochondrial networks in neurons by directly or indirectly regulating the activity of Drp1, other mitochondrial fission/ fusion (MFF) proteins and macroautophagy. ${ }^{8,14,34}$ Although the protective effects imparted by these two mitochondrially localized Ser/Thr kinases are similar, the observation that
PINK1 deficiency elicits such striking phenotypes despite normal levels of PKA suggests that they also have distinct functions at the level of mitochondria. Hence, we propose that PINK1 and PKA participate in parallel complementary signaling pathways to support mitochondrial and non-mitochondrial neuronal functions. The level of functional redundancy or compensation may be higher in some species, or more effective mitochondrial targeting of PKA may occur, as PINK1- or AKAP1-knockout mice do not elicit obvious brain degeneration. ${ }^{35,36}$

The possibility of PINK1 haploinsufficiency as a risk factor in sporadic PD has been raised but is controversial. Although haploinsufficiency does not induce overt neurodegeneration and PD, recent studies implicate molecular signaling alterations in dopaminergic neurons, including reduction in dopamine receptor content, neuropsychiatric abnormalities and slight motor dysfunctions. ${ }^{37}$ Cell culture studies have also demonstrated that transient overexpression of PD-associated mutations in PINK1 perturb mitochondrial morphology and calcium homeostasis, eliciting compensatory pathways such as macroautophagy. ${ }^{38}$ In the current study, heterozygous PINK1 cortical neurons exhibited nonsignificant trends towards increased mitochondrial fission, but no evidence of mitochondrial depolarization or of lysosomal expansion (Figure 5). In contrast, homozygous PINK1-knockout neurons, or acute RNAi-mediated knockdown of PINK1, not only led to mitochondrial fragmentation, but also to decreased mitochondrial membrane potential and lysosomal expansion as markers of stress/injury. The ameliorating effects of stimulating PKA were also observed in primary cortical neurons.

To summarize, we show for the first time that redirecting endogenous PKA to mitochondria via AKAP1 reverses mitochondrial dysfunction, macroautophagy, and cell death in PINK1 shRNA cell lines by stabilizing mitochondrial networks. Either increased mitochondrial localization of PKA activity, or modulation of the PKA phosphorylation site on Drp1, is sufficient to reverse alterations in mitochondrial structure and cell death (Figures $1 \mathrm{~d}$ and 4), reducing cellular signals to drive compensatory autophagy/mitophagy. Likewise, cAMP reverses fission, mitochondrial membrane potential, and lysosomal activation in primary cortical neurons. These data indicate that enhanced mitochondrial activities of PKA may be used to rescue mitochondrial deficits elicited by deficient PINK1 function, highlighting the importance of studying compartmentalized signaling networks in neurodegeneration and neuroprotection.

\section{Materials and Methods}

Plasmids. OMM-targeted GFP (yeast MAS70 pre-sequence), wild-type Drp1 and a set of GFP-tagged forms of rat Drp1 (S656A and S656D) were constructed in an EGFP-N1 backbone that co-express shRNA directed against all mammalian orthologs of Drp $1 .{ }^{14}$ GFP-tagged fusion constructs expressing the core domain (residues 1-524) of wild-type rat AKAP1 or a mutant form that is deficient in PKA binding $(\triangle-P K A ;$ I310P, and L316P) were sub-cloned to the pEGFP-C1 vector, as were GFP fusion constructs of untargeted and OMM-targeted PKA (hexokinasetargeting sequence) (Supplementary Figure 1b). GFP- and RFP-tagged constructs of LC3 (GFP-LC3 and RFP-LC3) were generously provided by Tamotsu Yoshimori (Graduate School of Biosciences and Frontier Bioscience, Osaka University, Suita-Osaka, Japan). ${ }^{21}$ The Mito-HyPer vector, which consists of a mitochondrially targeted, circularly permuted yellow fluorescent protein inserted into the regulatory 
domain of a bacterial hydrogen peroxide sensor, was purchased from Evrogen (Moscow, Russia).

Stable PINK1 shRNA knockdown cell lines. These stable SH-SY5Yderived lines were generated using two distinct shRNA sequences (A and D) as previously described. ${ }^{7}$ In some experiments, cells were treated with dibutyryl-cyclic AMP or forskolin (Sigma, St Louis, MO, USA) at a $250 \mu \mathrm{M}$ and $50 \mu \mathrm{M}$, respectively. SH-SY5Y cells were transfected with the DNA plasmids listed above using LipofectAMINE 2000 (Invitrogen, Carlsbad, CA, USA). For cells grown in four-well chambered coverslips or twelve-well plates, $1 \mu \mathrm{g}$ of DNA diluted in OPTIMEM was mixed with liposomes at a final concentration of $0.10 \%$.

Primary neuron cultures. Primary cortical neurons were prepared from 14day C57BL/6 mouse embryos (Hilltop Laboratory Animals, Inc., Scottdale, PA, USA) or from the PINK1 knockout mouse line developed from C57/129_PINK1 + / - ES cells by Dr. Chenjian Li (Mt. Sinai). The line is maintained as a heterozygous line; for primary culture studies, the embryos from a $+I-x+I-$ cross are individually harvested into separate wells and genotyped by PCR of DNA isolated from the tails. The neurons were seeded in poly-L-lysine-coated four-well chambered slides (Nunc Laboratory-Tek; Fisher Scientific, Agawam, MA, USA) at $3 \times 10^{5} \mathrm{cells} / \mathrm{cm}^{2}$ in serum-free Neurobasal media (Gibco, Invitrogen) and supplemented with $0.75 \mathrm{mM}$ L-glutamine (BioWhittaker, Walkersville, MD, USA), using a protocol approved by the University of Pittsburgh IACUC. After 3 days, two-thirds of media was changed with fresh Neurobasal media (Gibco, Invitrogen) with B27 supplement (NB/B27 media), $0.75 \mathrm{mM}$ L-glutamine. Small interfering RNA (siRNA) targeted against murine PINK1 were synthesized from Sigma and characterized as previously described. ${ }^{7}$ Because of low primary neuron transfection efficiency, the murine HT22 hippocampal cell line was transfected at $35 \%$ confluence with PINK1 siRNA ( $5^{\prime}$-CAAACACCUUGGCCUUAUA-3'), or equivalent siControl non-targeting siRNA (Dharmacon, Lafayette, CO, USA) $72 \mathrm{~h}$ before western blot confirmation of murine PINK1 knockdown.

Transmission electron microscopy. Cells grown in six-well tissue culture dishes were rinsed with phosphate-buffered saline (PBS) and fixed in $2.5 \%$ glutaraldehyde overnight at $4{ }^{\circ} \mathrm{C}$, processed, stained, and observed using a JEOL JEM 1210 transmission electron microscope (JEOL, Peabody, MA, USA) as previously described. ${ }^{7}$

Cell imaging. Live SH-SY5Y cells were stained with MitoTracker Green FM (Invitrogen, Molecular Probes) at a final concentration of $250 \mathrm{nM}$, incubated for $20 \mathrm{~min}$ at $37^{\circ} \mathrm{C}$ followed by a wash in regular media before imaging cells using an IDX71 Olympus fluorescence microscope at a magnification of $20 \times(0.45 \mathrm{NA})$ or $40 \times(0.60 \mathrm{NA})$ equipped with a DP70 microscope digital camera (Olympus America Inc., Melville, NY, USA; excitation/emission filter: 490/520 nm; 541/572 nm).

Western blot analysis and densitometry. Cell lysates (1.0\% Triton X-100 with protease/phosphatase inhibitors) were resolved on 5-15\% Ammediolbuffered polyacrylamide gels as previously described ${ }^{7}$ and immunoblotted for endogenous PINK1 using C8830 rabbit anti-PINK1 $(1: 2000),{ }^{7}$ or tagged proteins using mouse anti-Flag $(1: 1000)$ (Sigma), mouse anti-V5 tag $(1: 200-1: 500)$ or rabbit anti-GFP (1:1000) (Invitrogen), rabbit anti-Drp1 antibody (1:500; H300 Santa Cruz Biotechnology, Santa Cruz, CA, USA), mouse anti-phospho-Drp1 (PKA S656, rat; developed by Dr. Stefan Strack, University of lowa), anti-mouse PINK1 (Abgent, San Diego, CA, USA; AM6406a), with rabbit anti-GADPH $(1: 4000)$ or mouse anti- $\beta$-actin (1:1000) (Santa Cruz Biotechnology) as loading controls. Following overnight incubation, PVDF membranes were incubated with the appropriate secondary antibodies conjugated to horseradish peroxidase $(1: 5,000$; Jackson ImmunoResearch Laboratories, West Grove, PA, USA) in 5\% milk in PBST.

Cell survival-death assay. Cells were seeded on 24-well plates and transfected with GFP-tagged plasmids. At 2 days following transfection, cells were fixed in $4 \%$ paraformaldehyde, permeabilized in $0.1 \%$ Triton X-100 permeabilized and counterstained with DAPI ( $1.25 \mu \mathrm{g} / \mathrm{ml}$; Molecular Probes) for $5 \mathrm{~min}$ to visualize nuclei before washing two more times in PBS. Apoptosis was quantified as the percentage of transfected GFP cells containing fragmented or condensed nuclei or exhibiting cytosolic cytochrome $c$ (mouse anti-human cytochrome $c$; Santa Cruz Biotechnology, 1:1000) per epifluorescence micrograph, which were captured using an IDX71 Olympus fluorescence microscope at a magnification of $20 \times$
(Olympus America Inc.; excitation/emission filter: $490 / 520 \mathrm{~nm}$; $541 / 572 \mathrm{~nm}$ ) by an observer blinded to the experimental conditions.

Mitochondrial superoxide and mitochondrial hydrogen peroxide assays. At 2 days after transfection with PKA reagents, the cells were stained with MitoSOX, $\left(5 \mu \mathrm{M}\right.$, Molecular Probes) as previously described. ${ }^{7}$ The average intensity of MitoSOX fluorescence per cell in GFP-transfected cells was quantified using NIH Image $J$ software (National Institutes of Health, Bethesda, MD, USA), dividing the 'raw integrated density' of MitoSOX fluorescence by the mitochondrial area for each transfected cell. To measure intracellular hydrogen peroxide, cells were transfected with $0.5 \mu \mathrm{g}$ per well of Mito-Hyper vector for 2 days and cotransfected with a cytosolic RFP vector. The intracellular hydrogen peroxide levels in mitochondria were analyzed by acquiring single confocal slice scans using a Zeiss LSM confocal microscope (Carl Zeiss Microlmaging, Thornwood, NY, USA) as described above. The average mean fluorescence intensity/cell was analyzed using ImageJ (version 1.39).

Transmembrane potential assays. Control and PINK1 shRNA cells were treated with dibutyryl cAMP $(250 \mu \mathrm{M} \times 4 \mathrm{~h})$ or the $\mathrm{dH}_{2} \mathrm{O}$ vehicle. Cells were stained with the red/green fluorescent ratiometric JC-1 dye (Molecular Probes) at $2.5 \mu \mathrm{g} / \mathrm{ml}$ for $15 \mathrm{~min}$ at $37^{\circ} \mathrm{C}$ in the presence or absence of the mitochondrial uncoupler FCCP to assess the specific fluorescence contributed by transmembrane potential. After washing with PBS containing glucose $(4 \mathrm{~g} / \mathrm{l})$, transmembrane potential was quantified using the well-scan function (excitation wavelengths 490 and $533 \mathrm{~nm}$ and emission wavelengths of 525 and $590 \mathrm{~nm}$, respectively) of a Spectromax M2 microplate reader (Molecular Devices, Sunnyvale, CA, USA). The ratio of red to green fluorescence was quantified in eight to ten replicate wells per experiment following subtraction of background fluorescence in the presence of FCCP.

Primary neurons were loaded with $40 \mathrm{nM}$ of tetra-methyl rhodamine methylesther (TMRM) and incubated for $40 \mathrm{~min}$ at $37^{\circ} \mathrm{C}$ in a $5 \% \mathrm{CO}_{2}$ cell culture incubator followed by a single wash with fresh neurobasal medium supplemented with B27 and glutamax. The TMRM average pixel fluorescence intensity in mitochondria was normalized to the background fluorescence intensity measured within the nucleus. ${ }^{39}$

Quantification of GFP-LC3 puncta, mitophagy, and mitochondrial morphology. Cells were imaged for colocalization of RFP-LC3 puncta with green fluorescent labeled mitochondria (mitochondrially targeted GFP and PKA) using an inverted FluoView 1000 laser scanning confocal microscope at a 60X magnification (1.42 numerical aperture) using the linear, sequential scan-mode function (Olympus America Inc; excitation/emission filter: $488 / 510 \mathrm{~nm}$; $561 / 592 \mathrm{~nm}$ ) or with a Zeiss LSM 510 Meta laser-scanning confocal microscope (Carl Zeiss Microlmaging) (excitation/emission filter, 488/510 nm;561/592 nm). GFP-LC3 and RFP-LC3 puncta, colocalization of RFP-LC3 with MitoTracker green stained mitochondria (250 nM, Molecular Probes) or Lysotracker Red DND-99 (100 nM, Molecular Probes) particles were quantified as previously described, ${ }^{7}$ using a custom macro for $\mathrm{NIH}$ Image $\mathrm{J}$ software (1.39) Indices of mitochondrial interconnectivity were quantified using an $\mathrm{NIH}$ Image $\mathrm{J}$ macro that performs automated image analysis of individual mitochondrial particles as previously described. ${ }^{7}$

Mitochondrial respiration assay. Cellular respiration was analyzed using an XF24 Extracellular Flux Analyzer . Cells transiently transfected with GFP fusion constructs of PKA or treated with pharmacological activators of PKA (dibutyrylcyclic-AMP or forskolin) for $24 \mathrm{~h}$ were plated at a density of 100000 cells per well in DMEM media containing $10 \%$ fetal bovine serum and high glucose $(4 \mathrm{~g} / \mathrm{l}) 24 \mathrm{~h}$ before analysis. OCRs in living cells were analyzed at baseline and following the sequential addition of $1 \mu \mathrm{M}$ oligomycin, $300 \mathrm{nM} \mathrm{FCCP}, 120 \mathrm{mM}$ 2-deoxyglucose and $1 \mu \mathrm{M}$ rotenone, final concentrations.

CRE reporter activity assay. The effects of different GFP constructs of PKA on intracellular PKA activity were measured using the Clontech CRE-luciferase cisReporting System. (Stratagene, La Jolla, CA, USA). In brief, cells cultured on 24well plates were co-transfected with $0.25 \mu \mathrm{g} /$ well of a master mix containing Photinus firefly luciferase regulated by a CRE promoter with a constitutively active form of Renilla firefly luciferase as an internal transfection control at a 20:1 mass ratio of plasmids. At 2 days following transfection, cells were prepared and measured for luciferase activity as previously described. ${ }^{29}$ Light emitted from a $20 \mu \mathrm{l}$ of cell supernatant was monitored for $20 \mathrm{~s}$ in a Lumat LB9507 luminometer (Berthold Technologies, Oak Ridge, TN, USA). 
ATP assay. Cellular ATP concentrations were measured using the ATPLite-M luminescence assay (PerkinElmer; Waltham, MA, USA) and a Synergy 2 MultiMode Microplate Reader (BioTek, Winooski, VT, USA). Cells were plated at a density of 100000 per well in a black 96-well microplate and cultured in glucose-free DMEM containing galactose and glutamine $24 \mathrm{~h}$ before initiation of the experiment. Under these conditions, the cells rely solely on mitochondrial oxidative phosphorylation for ATP production. ${ }^{40}$ Luminescence readings were converted to ATP concentrations $(\mu \mathrm{M})$ based on a $0-10 \mathrm{M}$ standard curve.

Statistics. Unless otherwise indicated, results are expressed as mean \pm S.E.M. from at least three independent experiments. Multiple group comparisons were performed using one-way analysis of variance (ANOVA) followed by Tukey's test or Fisher's LSD. Values of $P<0.05$ were considered significant.

\section{Conflict of interest}

The authors declare no conflict of interest.

Acknowledgements. We thank Simon Watkins and Center for Biological Imaging (CBI) staff Mara Sullivan and Ming Sun at the University of Pittsburgh for assistance with EM imaging. This research was supported by the National Institutes of Health (AG026389, NS065789). CTC is recipient of an AFAR/Ellison Medical Foundation Julie Martin Mid-Career Award in Aging Research. RKD was supported in part by F32 AG030821 and by a Pathology Post-doctoral Research Training Program (PPRTP) grant (University of Pittsburgh).

1. Abeliovich A, Flint Beal M. Parkinsonism genes: culprits and clues. J Neurochem 2006; 99 1062-1072.

2. Valente EM, Salvi S, lalongo T, Marongiu R, Elia AE, Caputo V et al. PINK1 mutations are associated with sporadic early-onset parkinsonism. Ann Neurol 2004; 56: 336-341.

3. Samaranch L, Lorenzo-Betancor O, Arbelo JM, Ferrer I, Lorenzo E, Irigoyen J et al. PINK1-linked parkinsonism is associated with Lewy body pathology. Brain 2010; 133 (Part 4): 1128-1142.

4. Dagda RK, Zhu J, Chu CT. Mitochondrial kinases in Parkinson's disease: converging insights from neurotoxin and genetic models. Mitochondrion 2009; 9: 289-298.

5. Haque ME, Thomas KJ, D'Souza C, Callaghan S, Kitada T, Slack RS et al. Cytoplasmic Pink1 activity protects neurons from dopaminergic neurotoxin MPTP. Proc Natl Acad Sci USA 2008; 105: 1716-1721.

6. Wang HL, Chou AH, Yeh TH, Li AH, Chen YL, Kuo YL et al. PINK1 mutants associated with recessive Parkinson's disease are defective in inhibiting mitochondrial release of cytochrome c. Neurobiol Dis 2007; 28: 216-226.

7. Dagda RK, Cherra III SJ, Kulich SM, Tandon A, Park D, Chu CT. Loss of PINK1 function promotes mitophagy through effects on oxidative stress and mitochondrial fission. J Biol Chem 2009; 284: 13843-13855.

8. Sandebring A, Thomas KJ, Beilina A, van der Brug M, Cleland MM, Ahmad R et al. Mitochondrial alterations in PINK1 deficient cells are influenced by calcineurin-dependent dephosphorylation of dynamin-related protein 1. PLOS ONE 2009; 4: e5701.

9. Wood-Kaczmar A, Gandhi S, Yao Z, Abramov AS, Miljan EA, Keen G et al. PINK1 is necessary for long term survival and mitochondrial function in human dopaminergic neurons. PLOS ONE 2008; 3: e2455.

10. Poole AC, Thomas RE, Andrews LA, McBride HM, Whitworth AJ, Pallanck LJ. The PINK1/ Parkin pathway regulates mitochondrial morphology. Proc Natl Acad Sci USA 2008; 105 1638-1643.

11. Feliciello A, Gottesman ME, Avvedimento EV. The biological functions of A-kinase anchor proteins. J Mol Biol 2001; 308: 99-114.

12. Affaitati $A$, Cardone L, de Cristofaro T, Carlucci A, Ginsberg MD, Varrone $S$ et al. Essentia role of A-kinase anchor protein 121 for CAMP signaling to mitochondria. J Biol Chem 2003 278: 4286-4294.

13. Technikova-Dobrova Z, Sardanelli AM, Speranza F, Scacco S, Signorile A, Lorusso V et al. Cyclic adenosine monophosphate-dependent phosphorylation of mammalian mitochondrial proteins: enzyme and substrate characterization and functional role. Biochemistry 2001; 40: 13941-13947.

14. Cribbs JT, Strack S. Reversible phosphorylation of Drp1 by cyclic AMP-dependent protein kinase and calcineurin regulates mitochondrial fission and cell death. EMBO Rep 2007; 8 : 939-944.
15. Taguchi N, Ishihara N, Jofuku A, Oka T, Mihara K. Mitotic phosphorylation of dynamin-related GTPase Drp1 participates in mitochondrial fission. J Biol Chem 2007; 282: $11521-11529$.

16. Chang CR, Blackstone C. Cyclic AMP-dependent protein kinase phosphorylation of Drp1 regulates its GTPase activity and mitochondrial morphology. J Biol Chem 2007; 282: 21583-21587.

17. Han XJ, Lu YF, Li SA, Kaitsuka T, Sato Y, Tomizawa K et al. CaM kinase I alpha-induced phosphorylation of Drp1 regulates mitochondrial morphology. J Cell Biol 2008; 182: 573-585.

18. Cereghetti GM, Stangherlin A, Martins de Brito O, Chang CR, Blackstone $C$, Bernardi $P$ et al. Dephosphorylation by calcineurin regulates translocation of Drp1 to mitochondria. Proc Natl Acad SciUSA 2008; 105: 15803-15808.

19. Carlucci A, Lignitto L, Feliciello A. Control of mitochondria dynamics and oxidative metabolism by CAMP, AKAPs and the proteasome. Trends Cell Biol 2008; 18: 604-613.

20. Chu CT. A pivotal role for PINK1 and autophagy in mitochondrial quality control: implications for Parkinson disease. Hum Mol Genet 2010; 19 (R1): R28-R37.

21. Kimura S, Noda T, Yoshimori T. Dissection of the autophagosome maturation process by a novel reporter protein, tandem fluorescent-tagged LC3. Autophagy 2007; 3: 452-460.

22. Ginsberg MD, Feliciello A, Jones JK, Avvedimento EV, Gottesman ME. PKA-dependent binding of mRNA to the mitochondrial AKAP121 protein. J Mol Biol 2003; 327: 885-897.

23. Scherz-Shouval R, Shvets E, Fass E, Shorer H, Gil L, Elazar Z. Reactive oxygen species are essential for autophagy and specifically regulate the activity of Atg4. EMBO J 2007; 26: $1749-1760$

24. Exner N, Treske B, Paquet D, Holmstrom K, Schiesling C, Gispert S et al. Loss-of-function of human PINK1 results in mitochondrial pathology and can be rescued by parkin. J Neurosci 2007; 27: 12413-12418.

25. Liu W, Vives-Bauza C, Acin-Perez R, Yamamoto A, Tan Y, Li Y et al. PINK1 defect causes mitochondrial dysfunction, proteasomal deficit and alpha-synuclein aggregation in cell culture models of Parkinson's disease. PLoS ONE 2009; 4: e4597.

26. Rossignol R, Gilkerson R, Aggeler R, Yamagata K, Remington SJ, Capaldi RA. Energy substrate modulates mitochondrial structure and oxidative capacity in cancer cells. Cancer Res 2004; 64: 985-993.

27. Twig G, Elorza A, Molina AJ, Mohamed H, Wikstrom JD, Walzer G et al. Fission and selective fusion govern mitochondrial segregation and elimination by autophagy. EMBO J 2008; 27: 433-446.

28. Howells DW, Porritt MJ, Wong JY, Batchelor PE, Kalnins R, Hughes AJ et al. Reduced BDNF mRNA expression in the Parkinson's disease substantia nigra. Exp Neurol 2000; 166: $127-135$

29. Chalovich EM, Zhu JH, Caltagarone J, Bowser R, Chu CT. Functional repression of cAMP response element in 6-hydroxydopamine-treated neuronal cells. J Biol Chem 2006; 281 : 17870-17881.

30. Cherra III S, Kulich SM, Uechi G, Balasubramani M, Mountsouris J, Day BD et al. Regulation of the autophagy protein LC3 by phosphorylation. J Cell Biol 2010; 190: 533-539.

31. Deas E, Plun-Favreau H, Gandhi S, Desmond H, Kjaer S, Loh SH et al. PINK1 cleavage at position A103 by the mitochondrial protease PARL. Hum Mol Genet 2011; 20: 867-879.

32. Narendra DP, Jin SM, Tanaka A, Suen DF, Gautier CA, Shen J et al. PINK1 is selectively stabilized on impaired mitochondria to activate Parkin. PLoS biology 2010; 8: e1000298.

33. Amo T, Sato S, Saiki S, Wolf AM, Toyomizu M, Gautier CA et al. Mitochondrial membrane potential decrease caused by loss of PINK1 is not due to proton leak, but to respiratory chain defects. Neurobiol Dis 2011; 41: 111-118.

34. Cui M, Tang X, Christian WV, Yoon Y, Tieu K. Perturbations in mitochondrial dynamics induced by human mutant PINK1 can be rescued by the mitochondrial division inhibitor mdivi-1. J Biol Chem 2010; 285: 11740-11752.

35. Gautier CA, Kitada T, Shen J. Loss of PINK1 causes mitochondrial functional defects and increased sensitivity to oxidative stress. Proc Natl Acad Sci USA 2008; 105: 11364-11369.

36. Newhall KJ, Criniti AR, Cheah CS, Smith KC, Kafer KE, Burkart AD et al. Dynamic anchoring of PKA is essential during oocyte maturation. Curr Biol 2006; 16: 321-327.

37. Guo JF, Wang L, He D, Yang QH, Duan ZX, Zhang XW et al. Clinical features and [(11)C]CFT PET analysis of PARK2, PARK6, PARK7-linked autosomal recessive early onset Parkinsonism. Neurol Sci 2011; 32: 35-40.

38. Marongiu R, Spencer B, Crews L, Adame A, Patrick C, Trejo M et al. Mutant Pink1 induces mitochondrial dysfunction in a neuronal cell model of Parkinson's disease by disturbing calcium flux. J Neurochem 2009; 108: 1561-1574.

39. Dagda RK, Barwacz CA, Cribbs JT, Strack S. Unfolding-resistant translocase targeting: a novel mechanism for outer mitochondrial membrane localization exemplified by the Bbeta2 regulatory subunit of protein phosphatase 2 A. J Biol Chem 2005; 280: 27375-27382.

40. Reitzer LJ, Wice BM, Kennell D. Evidence that glutamine, not sugar, is the major energy source for cultured HeLa cells. J Biol Chem 1979; 254: 2669-2676.

\section{Supplementary Information accompanies the paper on Cell Death and Differentiation website (http://www.nature.com/cdd)}

